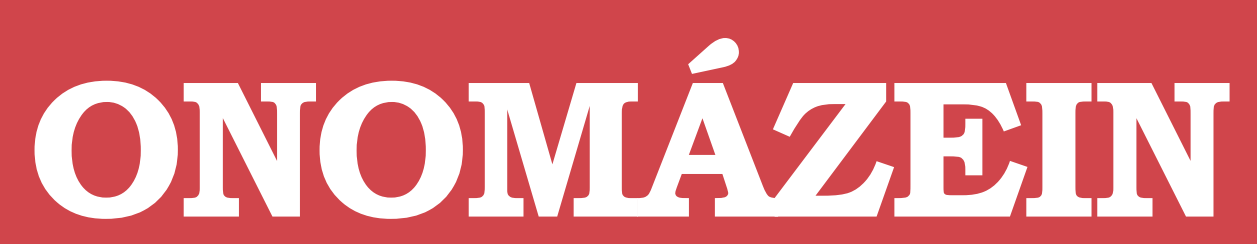

Revista de lingüística, filología y traducción
PONTIFICIA UNIVERSIDAD

\title{
Memoria operativa y complejidad cognitiva en tareas de expresión escrita en español como lengua extranjera
}

Working memory and cognitive complexity of writing tasks in Spanish as a foreign language

\author{
Irini Mavrou \\ Universidad Antonio de Nebrija \\ España
}

ONOMÁZEIN 41 (septiembre de 2018): 125-146

DOI: 10.7764/onomazein.41.02

\section{(c) $($ i $)$}

Irini Mavrou: Departamento de Lenguas Aplicadas y Educación, Universidad Antonio de Nebrija, España. | Correo electrónico: emavrou@nebrija.es 


\section{Resumen}

El objetivo del presente estudio fue examinar la incidencia de la memoria operativa en la producción escrita de 65 aprendientes griegos de español como lengua extranjera. Los informantes realizaron dos tareas, la primera bajo presión de tiempo y la segunda bajo condiciones relativamente más favorables de actuación, y sus textos se evaluaron mediante una escala de evaluación analítica. La capacidad de la memoria operativa se midió a través de las pruebas Speaking Span Task y Math Span Task. Asimismo, se utilizó una prueba de repetición de pseudopalabras para evaluar su capacidad de la memoria fonológica. Los resultados mostraron que la memoria operativa predijo de manera significativa la calidad del discurso escrito, siendo su contribución ligeramente mayor en la tarea de mayor complejidad cognitiva. Por otro lado, la memoria fonológica tuvo una influencia positiva en la actuación, aunque solo en la realización de la tarea de menor complejidad cognitiva.

Palabras clave: memoria operativa; memoria fonológica; complejidad cognitiva; producción escrita.

\section{Abstract}

The purpose of this study was to investigate the role of working memory capacity (WMC) in foreign language (FL) written production. Sixty-five Greek learners of Spanish as a FL performed two written tasks, the first one under time pressure and the second one under relatively easier task conditions (i.e. more time-on-task), and their linguistic performance was assessed by an analytical assessment scale. WMC was measured by means of three tasks, i.e. the Speaking Span Task, the Math Span Task and a non-word repetition task, which was designed in order to measure the phonological short-term memory capacity. The results showed that WMC made a statistically significant contribution to the prediction of text quality in both tasks, which was slightly higher in the performance of the more complex task, while phonological short-term memory capacity had a positive influence only on the performance of the less demanding task.

Keywords: working memory; phonological short-term memory; cognitive complexity; written production. 


\section{Introducción}

En el ámbito de la psicología cognitiva, donde el estudio de la memoria ha ocupado un lugar prioritario, se ha prestado especial atención a un componente de esta, la memoria operativa (MO). Este mecanismo permite almacenar temporalmente y ensayar mentalmente la información, supervisar y controlar la distribución de los recursos atencionales, activar conocimiento de la memoria a largo plazo (MLP), mantener esta activación e inhibir la interferencia generada por la activación de información irrelevante (Baddeley y Logie, 1999; Engle, Kane y Tuholski, 1999; Kane, Conway, Hambrick y Engle, 2007; Unsworth y Engle, 2007b). Como sostiene Andrade (2001: 3), comprender estos procesos es fundamental para la comprensión de casi todos los demás aspectos de la cognición.

A pesar de que la MO ha llegado a convertirse en un constructo de suma importancia también en el ámbito de adquisición de segundas lenguas (ASL) y ha sido objeto de estudio de un amplio número de investigaciones, su contribución en los procesos de producción escrita en una lengua extranjera (LE) se ha abordado de manera escasa y fragmentaria (Mavrou, 2017). Como señala Kormos (2012: 390), "although the importance of individual differences, such as working memory capacity, motivation, and self-efficacy beliefs, has been explored in many studies in the field of LI writing research ..., little is known about how learner differences affect L2 writing processes".

Lograr un buen dominio de la producción escrita, sea en la lengua materna (LM) o en una LE, no es un proceso automático, sino que requiere práctica, entrenamiento, instrucción (Kellogg, 2008). Según Kellogg (1986), el esfuerzo cognitivo que requieren los procesos de escritura, su competición por los recursos cognitivos limitados del individuo y su interacción con factores contextuales y afectivos convierten la escritura en la LM en una tarea mucho más exigente que otras actividades humanas. Si esto es cierto para el caso de la LM, sería razonable asumir que escribir en una LE supondría niveles de dificultad aún más altos y, probablemente, un consumo devastador de los recursos cognitivos del aprendiente.

Considerando la escasez de referentes actuales en el ámbito de ASL sobre la incidencia de la MO en la producción de textos escritos, así como la advertencia de muchos autores de intentar superar "el sesgo monolingüe, monocultural y etnocéntrico" que había supuesto durante muchos años la investigación empírica y las teorías de composición escrita basadas casi exclusivamente en la escritura en inglés (Silva, Leki y Carson, 1997; Cumming, 2009; Manchón, 2009; Ortega, 2009), el presente estudio intenta profundizar en la relación entre la MO y la expresión escrita en español como LE (ELE) de un grupo de hablantes nativos de griego. Asimismo, partiendo del marco cognitivo-interaccionista (Robinson, 2001, 2003; Skehan y Foster, 2001), se examina dicha relación a partir de dos tareas narrativas que difieren en su complejidad cognitiva, manipulándose la cantidad de tiempo para su ejecución [^time-on-task], 
una variable especialmente relevante para los procesos de escritura, aunque desatendida en los estudios que se adscriben a la línea de manipulación cognitiva de tareas.

\section{Metodología 2.1. Participantes}

En el estudio participaron 65 aprendientes griegos de ELE del Instituto Cervantes de Atenas y del Centro de Idiomas Extranjeros de la Universidad Kapodistríaca (Atenas), 16 hombres (24.6\%) y 49 mujeres (75.4\%), de edades comprendidas entre los 20 y los 38 años $(M=26.26$, $S D=4.94$, Mediana=24). Todos los informantes asistían a cursos de nivel intermedio (B2), habían estudiado el español durante un promedio de 2.54 años académicos y eran hablantes de aproximadamente tres LE, incluido el español. En concreto, a excepción de un caso, todos habían estudiado el inglés durante un promedio de 8.41 años (Rango=5-13 años), 35 de ellos también el francés ( $M=5.54$; Rango=1.5-10 años) y con menor frecuencia otras LE como el alemán ( $n=6$; $M=6.83 ;$ Rango=3-17) y el italiano ( $n=7 ; M=1.93 ;$ Rango=0.5-3). Además, 32 informantes (49.2\%) habían estado en países de habla hispana por diversas razones (vacaciones, estudios, trabajo y visitas a amigos) durante un periodo de tiempo inferior a los 6 meses para un porcentaje acumulado del 41.5\%, mientras que el resto (50.8\%) no había visitado nunca países hispanohablantes (para una descripción más detallada de los informantes véase Mavrou, 2018).

\subsection{Instrumentos}

\subsubsection{Pruebas de memoria}

La capacidad de la MO de los informantes fue evaluada a partir de las pruebas Speaking Span Task y Math Span Task. Además, se utilizó una prueba de repetición de pseudopalabras con el objetivo de medir la capacidad del componente fonológico de la MO.

El Speaking Span Task (SST) se basó en una adaptación parcial al griego de la prueba que utilizaron Prebianca y D’Ely (2008: 361-362). Para su construcción, se establecieron a priori las siguientes pautas: (1) incluir solamente palabras bisílabas y trisílabas; (2) evitar el uso, dentro del mismo conjunto, de palabras semántica y fonológicamente relacionadas; (3) homogeneidad en cuanto a la forma (solo palabras en singular).

EI SST, en su versión final, estuvo compuesto por cinco niveles, del 2 al 6, cada uno de los cuales contenía tres conjuntos de palabras, de longitud ortográfica de entre 4 y 8 letras, y cuyo número iba en aumento en función del nivel de dificultad de la prueba. Su administración fue digitalizada mediante el programa PowerPoint. Las palabras se presentaban con una frecuencia de aparición de 1 segundo y, una vez presentado cada conjunto de palabras, los in- 
formantes tenían que producir oralmente tantas frases como fuera posible utilizando dichas palabras. Si bien no hubo restricciones en cuanto a la complejidad de las oraciones o a su longitud, se les explicó que se considerarían correctas solo aquellas oraciones que fueran aceptables en sus aspectos gramaticales y semánticos (Mota, 2003; Prebianca y D’Ely, 2008). Asimismo, se les informó que se penalizaría la repetición del mismo patrón de oración con palabras pertenecientes al mismo conjunto (Mizera, 2006). Con ello se intentó impedir el apoyo en patrones repetitivos o memorizados que pudieran disminuir la carga cognitiva (Mizera, 2006).

El tiempo concedido para la producción de oraciones fue determinado por la cantidad de estas, es decir, para la producción de dos frases (nivel 2) los informantes disponían de 20 segundos, para la producción de tres frases (nivel 3) el tiempo aumentaba en 30 segundos y así sucesivamente (esto es, 10 segundos para cada oración). La actuación de los informantes en el SST se evaluó de la siguiente manera: por cada palabra recuperada en su posición serial exacta y utilizada en una frase aceptable se asignaba un punto, mientras que si el informante producía frases aceptables, pero sin respetar el orden de aparición de las palabras o cambiando la forma o el número de estas (de singular a plural), se le concedía medio punto. La máxima puntuación que se podía obtener era de 60 puntos.

La segunda prueba de MO fue el Math Span Task (MST). El MST fue tomado de ShahnazariDorcheh y Roshan (2012) y comprendió un total de 60 ecuaciones matemáticas simples, 30 de adición y 30 de sustracción, distribuidas en cinco niveles (del 2 al 6), cada uno de los cuales contenía tres conjuntos de entre 2 y 6 problemas matemáticos. Su administración también fue informatizada, elaborada en PowerPoint. En el centro de la pantalla de un ordenador portátil aparecía un problema matemático del tipo $\mid X+Y=$ ?| o |X $-Y=$ ?|, donde $X$ e $Y$ se corresponden con números del 1 al 9. Los informantes, además de proporcionar oralmente la solución al problema, tenían que retener el segundo dígito de cada problema matemático para su posterior recuperación (en las fórmulas $|X+Y|$ y $|X-Y|$ el dígito meta sería el $Y$ ).

Con el objetivo de impedir la estrategia de repetición subvocal se establecieron unos límites temporales. En concreto, cada problema matemático permanecía a la vista durante 3 segundos y, una vez presentado cada conjunto de problemas matemáticos, los participantes disponían de ente 4 y 12 segundos para recuperar los dígitos meta. El tiempo fue determinado por el número de dígitos que tenían que recuperarse (dos segundos por cada dígito). La amplitud [span] de MO a partir del MST se estimó en función del número total de dígitos meta correctamente recuperados y en su posición serial exacta, siempre que se resolviera correctamente el problema matemático al que pertenecían. La máxima puntuación era también de 60 puntos.

La tercera prueba de memoria fue la de repetición de pseudopalabras (PRP). Para su construcción se adoptaron las siguientes pautas: (1) crear pseudopalabras que respetaran las características del idioma griego en términos de entonación y combinación entre fonemas (Masoura, Gathercole y Bablekou, 2004); las combinaciones de fonemas que se utilizaron 
fueron |V|, |VC|, |CV|, |CVC|, |CCV|, |CCCV|, donde C se corresponde con una consonante y V con una vocal (Maridaki-Kassotaki, 1998); (2) controlar, en la medida de lo posible, el efecto de wordlikeness, que se refiere al grado de similitud de una pseudopalabra con otra palabra real, almacenada en el léxico mental del individuo (Gathercole, 1995; Masoura y otros, 2004; BreaSpahn, 2009); (3) incluir pseudopalabras cuya sílaba tónica no se corresponda con una palabra existente en el idioma griego (Dollaghan, 1987, en Masoura y otros, 2004: 345).

La PRP, en su versión final, constó de cinco niveles (del 2 al 6), cada uno de los cuales contenía tres listas de pseudopalabras bisílabas, de longitud ortográfica de entre 4 y 6 letras, y cuyo número iba en aumento de acuerdo con el nivel de dificultad de la prueba. De este modo, en el nivel 2 hubo tres listas de dos pseudopalabras, el nivel 3 incluyó tres listas de tres pseudopalabras cada una y así sucesivamente. Durante la administración de la prueba la investigadora leía las pseudopalabras a un ritmo constante de aproximadamente I segundo por palabra, procurando que la prosodia fuera normal, y después el informante tenía que repetirlas oralmente en el orden de presentación. El span de memoria fonológica se obtuvo sumando el número total de pseudopalabras correctamente recuperadas y en su posición serial exacta (máxima puntuación: 60 puntos).

\subsubsection{Tareas escritas}

El corpus de producciones escritas se generó a partir de dos tareas narrativas basadas en material gráfico. La manipulación de su complejidad cognitiva se hizo en función del tiempo que dispondrían los informantes para su realización [^time-on-task]. En concreto, para la primera tarea (T1) se concedieron 10 minutos, mientras que para la segunda (T2) 20 minutos, aunque en ambos casos se les pidió a los informantes que redactaran sus textos utilizando los tiempos verbales del pasado (para una descripción más detallada de las tareas véase Mavrou y Villar, 2017).

\subsection{Procedimiento de recogida de datos}

La obtención de las muestras escritas se hizo en los correspondientes centros de estudio de ELE. Con el objetivo de controlar el efecto de práctica y anular los efectos secuenciales (orden de realización de las tareas), se aplicó la técnica de contrabalanceo entresujeto de la siguiente manera: se comenzó con el orden T1T2 en el primer grupo (clase intacta), se invirtió este orden en el siguiente grupo (T2T1) y así sucesivamente. La administración de las pruebas de memoria se llevó a cabo fuera de clase y de manera individual. Las citas se concentraron en un lugar previamente elegido por los propios informantes y a una hora que les resultara favorable, aunque en la mayoría de los casos se establecieron por la tarde, en el centro de estudios al que asistían y tuvieron una duración de aproximadamente 45-60 minutos. 


\subsection{Evaluación de la expresión escrita}

La actuación de los informantes en las dos tareas se evaluó mediante una escala analítica que fue tomada de Eguiluz Pacheco y Eguiluz Pacheco (2004). Dado que dicha escala había sido diseñada para un contexto de evaluación certificativa, se sometió a un pilotaje con el objetivo de detectar y reparar posibles problemas que pudieran surgir al ser aplicada en un contexto de evaluación diferente.

Siguiendo las recomendaciones de Wang (2009), en una primera etapa se transcribieron los datos, se seleccionó un número reducido de composiciones ( $n=14 ; 7$ de la T1 y 7 de la T2) que representaban textos de diferente calidad, y se entregaron en orden aleatorio a dos profesoras, ambas doctoras en Lingüística Aplicada a la enseñanza de ELE, que evaluaron los textos de modo ciego. El pilotaje condujo a algunas ligeras modificaciones que se introdujeron en la escala original con el objetivo de acomodarla a los objetivos del estudio.

Los descriptores de la escala fueron cuatro: (1) adecuación (tipo de documento, situación comunicativa, cumplimiento de las instrucciones proporcionadas); (2) estructura (organización de ideas e interrelaciones entre las distintas partes del discurso); (3) corrección gramatical (errores morfosintácticos y ortográficos); (4) vocabulario (variedad y precisión léxicas). La máxima puntuación que podía recibir una muestra escrita era de 15 puntos.

La evaluación final de todos los textos se hizo por la propia investigadora (EVI) y una de las profesoras que participó en el pilotaje de la escala (EV2), mientras que la puntuación final se derivó del promedio de las dos puntuaciones otorgadas. La fiabilidad de la escala fue satisfactoria tanto para la T1 (Cronbach's $\alpha_{\left(\mathrm{EV}_{1}\right)}=.850$ y Cronbach's $\alpha_{\left(\mathrm{EV}_{2}\right)}$ $=.818)$ como para la T2 (Cronbach's $\alpha_{(\mathrm{EV} 1)}=.831$ y Cronbach's $\left.\alpha_{(\mathrm{EV} 2)}=.752\right)$. Además, todas las correlaciones ítem-total corregidas fueron mayores que .40. En cuanto a la confiabilidad interevaluador, se estimó utilizando el coeficiente de correlación intraclase (CCI) (modelo matemático de efectos mixtos, acuerdo absoluto, medidas individuales) y fue $\mathrm{CCl}=.812$, 95\%IC [.705, .882] para la T1, y CCI=.843, 95\%IC $[.645, .920]$ para la T2. De acuerdo con las directrices propuestas por Cicchetti (1994, en Hallgren, 2012), el grado de confiabilidad interevaluador resultó alto ( $\mathrm{CCl}>$.75).

\section{Resultados}

\subsection{Consistencia interna de las pruebas de memoria}

La consistencia interna de las pruebas de memoria se examinó a través del coeficiente $\alpha$ de Cronbach y tras adoptar un procedimiento análogo al empleado en otras investigaciones sobre MO (Turner y Engle, 1989; Engle, Cantor y Carullo, 1992; Mota, 2003). En concreto, la es- 
timación de dicho coeficiente se basó en tres puntajes compuestos que se correspondían con la suma de aciertos en los primeros, segundos y terceros conjuntos de ítems de todos los niveles de cada prueba. Para aquellas pruebas que comprendían ítems lingüísticos, es decir, la PRP y el SST, el coeficiente de Cronbach también se calculó en función del número total de elementos (6o ítems en cada prueba), mientras que para el MST se estimó para los componentes de procesamiento (resolución de los problemas matemáticos) y de almacenamiento (recuperación de los dígitos meta) por separado, al igual que en el estudio de ShahnazariDorcheh y Roshan (2012).

\section{TABLA 1}

Consistencia interna de las pruebas de memoria

\begin{tabular}{lcccc}
\hline & $\begin{array}{c}\text { Alfa de } \\
\text { Cronbach }\end{array}$ & $\begin{array}{c}\text { Alfa de Cronbach } \\
\text { (60 elementos) }\end{array}$ & $\begin{array}{c}\text { Alfa de Cronbach } \\
\text { componente de procesamiento }\end{array}$ & $\begin{array}{c}\text { Alfa de Cronbach } \\
\text { componente de almacenamiento }\end{array}$ \\
\hline SST & .669 & .678 & - & - \\
MST & .882 & - & .709 & .858 \\
PRP & .801 & .746 & - & - \\
\hline
\end{tabular}

Como se puede apreciar en la tabla 1 , el rango de los coeficientes obtenidos fue entre .669 y .858. Teniendo en cuenta los valores de consistencia interna hallados en estudios previos, las directrices de varios autores (Streiner, 2003: 103; Field, 2009 [2000]: 657), así como la naturaleza exploratoria de la presente investigación, se puede concluir que la magnitud de dichos coeficientes fue satisfactoria.

\subsection{Memoria operativa y expresión escrita}

La tabla 2 presenta los valores descriptivos de las variables examinadas: span de memoria operativa (MST, SST), span de memoria fonológica (PRP) y puntuación final en las T1 y T2 (T1EvGlobal y T2EvGlobal). Como se observa, los valores de asimetría y de curtosis estuvieron dentro del intervalo $|-1+1|$, y los correspondientes a estos estadísticos puntajes tipificados (z-scores de asimetría y de curtosis) fueron menores que 1.96, por lo que se puede asumir distribución muestral de la media aproximadamente normal para todas las variables (Field, 2009 [2000]).

La tabla 3 comprende los coeficientes de correlación de Pearson entre la capacidad de la memoria y las T1EvGlobal y T2EvGlobal. Como se puede apreciar, las correlaciones entre el MST y las T1EvGlobal ( $r=.388$, $p=.001)$ y T2EvGlobal $(r=.356, p=.002)$ fueron positivas y estadísticamente significativas. Las obtenidas entre el SST y las T1EvGlobal ( $r=.194, p=.061)$ y T2EvGlobal $(r=.194, p=.061)$, aunque positivas, no resultaron significativas al nivel .05, mientras que el span 
de memoria fonológica correlacionó solo con la T2EvGlobal $(r=.302, p=.007)^{1}$. La aplicación del coeficiente de correlación parcial con variable de control la edad (tabla 3) arrojó resultados casi idénticos, lo cual permite afirmar que la edad no se convirtió en una variable confound.

A continuación, se efectuaron dos modelos de regresión lineal, el primero con variable predictora el MST y variable respuesta la TıEvGlobal (tabla 4), y el segundo con variables predictoras el MST, que se introdujo en el primer paso, y la PRP, que se añadió en el segundo paso, y variable respuesta la T2EvGlobal (tabla 5).

\section{TABLA 2}

Estadísticos descriptivos del span de memoria y de la puntuación final en las T1 y T2

\begin{tabular}{lccccc}
\hline & MST & SST & PRP & T1EvGlobal & T2EvGlobal \\
\hline Media & 33.09 & 33.42 & 35.82 & 9.54 & 9.92 \\
SD & 8.69 & 5.25 & 6.19 & 2.14 & 1.96 \\
Asimetría & 0.24 & 0.40 & 0.16 & 0.41 & 0.13 \\
SE & .30 & .30 & .30 & .30 & .30 \\
Curtosis & -0.61 & -0.56 & -0.84 & -0.69 & -0.90 \\
SE & .59 & .59 & .59 & .59 & .59 \\
\hline
\end{tabular}

\section{TABLA 3}

Correlaciones entre el span de memoria y las TIEvGlobal y T2EvGlobal

\begin{tabular}{|c|c|c|c|c|}
\hline & & MST & SST & PRP \\
\hline $\begin{array}{l}\text { T1EvGlobal } \\
\text { T2EvGlobal }\end{array}$ & & $\begin{array}{l}.388^{* * *} \\
.356^{* *}\end{array}$ & $\begin{array}{l}.194 \\
.194\end{array}$ & $\begin{array}{l}.175 \\
.302^{* *}\end{array}$ \\
\hline $\begin{array}{l}\text { Variable de control } \\
\text { Edad }\end{array}$ & $\begin{array}{l}\text { T1EvGlobal } \\
\text { T2EvGlobal }\end{array}$ & $\begin{array}{l}.388^{* \star *} \\
.356^{* *}\end{array}$ & $\begin{array}{l}.198 \\
.196\end{array}$ & $\begin{array}{l}.149 \\
.295^{\star *}\end{array}$ \\
\hline
\end{tabular}

Según los resultados del primer modelo (tabla 4), el MST dio cuenta de un 15\% de la variabilidad en la TıEvGlobal. Dado que el tamaño muestral no fue grande, se hizo también un ajuste y se obtuvo un porcentaje aproximado del $13.7 \%$. Los resultados del ANOVA fueron significativos $(F(1,63)=11.13, p=.001)$, lo cual indica que el modelo fue válido y resultó en un buen grado de predicción de la variable dependiente. Además, el valor del coeficiente de regresión fue

1 Debe aclararse que se llevaron a cabo procedimientos de contraste de hipótesis unilaterales, puesto que se partió de la hipótesis de que aquellos aprendientes con alta capacidad de MO obtendrían una mayor puntuación en las Tı y T2. 
significativamente distinto de cero $(\beta=.39, t(63)=3.34, p=.001)$; por tanto, se puede concluir que el span de MO contribuyó de manera relevante a la predicción de la TıEvGlobal. Se detectó solo un caso con residuo tipificado igual a 2.68 (caso 50), siendo todos los demás residuos tipificados menores que |1.96|, de ahí que el nivel de error en el modelo se considerara aceptable (Field, 2009 [2000]: 216). Al efectuarse el mismo modelo de regresión después de eliminar este caso, se observó un aumento en el porcentaje de la variación explicada $\left(R^{2}=.195, R^{2}\right.$ corregi$\mathrm{da}=.182, F(1,62)=14.99, p<.001)$, así como en el valor del coeficiente beta $(B=.44)$.

\section{TABLA 4}

Regresión lineal simple de la contribución de la memoria (MST) a la TıEvGlobal

\begin{tabular}{|c|c|c|c|c|c|c|c|c|}
\hline & $R^{2}$ & $\begin{array}{c}R^{2} \\
\text { Corregida }\end{array}$ & $F$ & $B$ & $S E B$ & $\beta$ & \multicolumn{2}{|c|}{$95 \%$ IC para $B$} \\
\hline Constante & & & & 6.38 & 0.98 & & 4.43 & 8.34 \\
\hline MST & .150 & .137 & $11.13^{* * *}$ & 0.10 & 0.03 & $.388^{* * *}$ & 0.04 & 0.15 \\
\hline
\end{tabular}

\section{TABLA 5}

Regresión lineal jerárquica de la contribución de la memoria (MST, PRP) a la T2EvGlobal

\begin{tabular}{lccccccccc}
\hline & $R^{2}$ & $\begin{array}{c}R^{2} \\
\text { Cor. }\end{array}$ & \multicolumn{1}{c}{$\Delta R^{2}$} & $F$ & $B$ & $\begin{array}{c}S E \\
B\end{array}$ & $\beta$ & \multicolumn{2}{c}{$\begin{array}{c}95 \% \text { IC } \\
\text { para } B\end{array}$} \\
\hline Paso 1 & & & & & & & & & \\
(Constante) & .127 & .113 & $.127^{* *}$ & $9.17^{* *}$ & 7.26 & 0.91 & & 5.45 & 9.07 \\
$\quad$ MST & & & & & 0.08 & 0.03 & $.356^{* *}$ & 0.03 & 0.13 \\
Paso 2 & & & & & & & & & \\
(Constante) & & & & & 5.15 & 1.43 & & 2.28 & 8.01 \\
MST & .174 & .148 & .047 & $6.55^{* *}$ & 0.07 & 0.03 & $.298^{*}$. & 0.01 & 0.12 \\
PRP & & & & & 0.07 & 0.04 & 225 & -0.004 & 0.15 \\
\hline
\end{tabular}

${ }^{*} p \leq .05,{ }^{* *} p \leq .01, D W=1.674$

En lo que se refiere al segundo modelo (tabla 5), el span de memoria (MST y PRP) predijo de manera significativa la actuación de los informantes en la T2, dando cuenta de un $17.4 \%$ de la variabilidad en la T2EvGlobal $(F(2,62)=6.55, p=.003)$, porcentaje que presentaría una disminución del 2.6\% si el modelo se derivara de la población. Más precisamente, el MST tuvo un efecto principal, explicando un $12.7 \%$ de la variabilidad en la T2EvGlobal $(F(1,63)=9.17, p=.004)$. La inclusión de la PRP en el modelo produjo un incremento de la varianza explicada de $\Delta R 2=.05$, aunque este cambio no resultó significativo. En cuanto a los valores de los coeficientes beta, solo los correspondientes al MST fueron significativamente distintos de cero (Paso 1: $B=.36$; Paso 2: $B=.30$ ). De nuevo se detectó un caso con residuo tipificado superior a 2.5, que pertene- 
cía al mismo informante que antes (caso 50), probablemente debido a que la actuación de este informante en ambas tareas escritas fue casi perfecta, mientras que su span de Mo fue relativamente bajo (MST=24). Además, no se detectaron datos extremos en el espacio definido por las variables predictoras², y el valor del estadístico de Durbin-Watson ( $D W=1.67$ ) fue superior a los valores críticos $d_{L}=1.54$ y $d_{u}=1.66$ para $n=65, k=2$ y $\alpha=.05$ (Kutner, Nachtsheim, Neter y Li, 2005 [1974]), lo cual indica la ausencia de autocorrelación en los residuos. Al efectuarse el mismo modelo después de eliminar el caso 50, el porcentaje de la variación explicada alcanzó el $24.4 \%(F(2,61)=9.86, p<.001)$. El MST dio cuenta de un $16.7 \%$ de la variabilidad en la T2EvGlobal, y la PRP contribuyó con un porcentaje adicional del $7.7 \%$, el cual fue significativo $(\triangle F(1,61)=6.23$, $p=.015)$. En cuanto a los valores de los coeficientes de regresión, fueron significativamente distintos de cero tanto para el MST (Paso 1: $\beta=.41$; Paso 2: $\beta=.34$ ) como para la PRP ( $\beta=.29$ ).

Teniendo en cuenta los resultados arrojados, sobre todo la inestabilidad que se produjo en cuanto a la contribución de la memoria fonológica a la T2EvGlobal, se llevó a cabo un procedimiento de validación cruzada. Para ello se seleccionó un subgrupo de la muestra final ( $n=42)$ lo más homogéneo posible respecto a los años de instrucción formal en ELE (2-3 años) y la duración de estancia en países de habla hispana (máximo: 3 meses) y se volvieron a efectuar los mismos modelos de regresión. Como se puede apreciar en las tablas 6 y 7, el MST dio

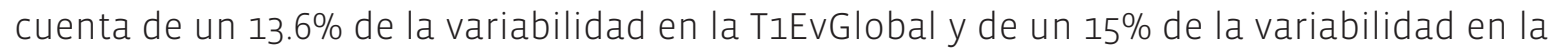
T2EvGlobal. La PRP, a pesar de contribuir con un aumento del $5.8 \%$ a la variabilidad observada en la T2EvGlobal, tuvo una capacidad explicativa menor $(B=.25)$.

\section{TABLA 6}

Regresión lineal simple de la contribución de la memoria (MST) a la TıEvGlobal (n=42)

\begin{tabular}{|c|c|c|c|c|c|c|c|c|}
\hline & $R^{2}$ & $\begin{array}{c}R^{2} \\
\text { Corregida }\end{array}$ & $F$ & $B$ & $S E B$ & $\beta$ & \multicolumn{2}{|c|}{$95 \%$ IC para $B$} \\
\hline Constante & & & & 6.64 & 1.27 & & 4.08 & 9.20 \\
\hline MST & .136 & .114 & $6.28^{*}$ & 0.09 & 0.04 & $.368^{*}$ & 0.02 & 0.16 \\
\hline
\end{tabular}

\section{Discusión}

El objetivo del presente estudio fue examinar el impacto de la MO en la producción escrita de un grupo de aprendientes griegos de ELE. Para ello se utilizaron dos tareas que diferían en su complejidad cognitiva, manipulándose el tiempo concedido para su realización. Los resulta-

2 El máximo valor de la distancia de Mahalanobis fue 6.38, el cual es menor que el valor crítico $D^{2}=12.23$ para $k=2, n=50$ y $\alpha=.05$ (Stevens, 1984). 


\section{TABLA 7}

Regresión lineal jerárquica de la contribución de la memoria (MSTGR, PRPGR) a la T2EvGlobal ( $n=42)$

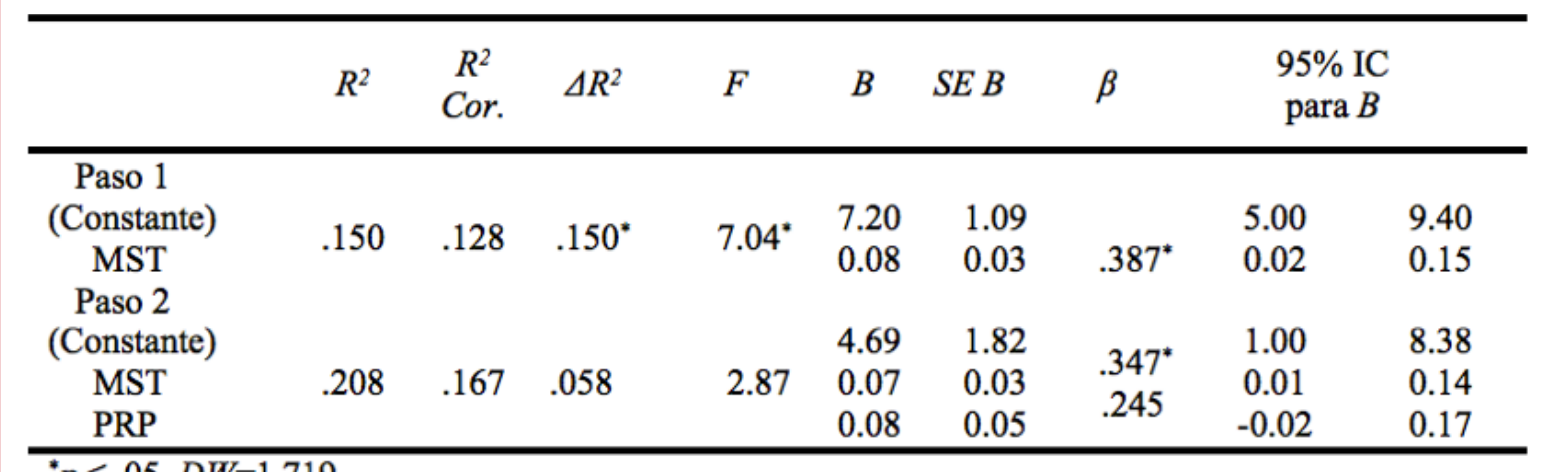

dos mostraron que la capacidad de la MO predijo de manera significativa la actuación de los informantes, independientemente de la complejidad cognitiva de las tareas implementadas.

Se podría asumir que el material gráfico condujo al desencadenamiento, activación y transferencia, de la MLP a la MO, de cierto vocabulario y de conocimiento declarativo relacionado con las propiedades gramaticales de la lengua meta, necesarios para convertir lo que “cuentan” las imágenes en output escrito. Según Unsworth y Engle (2007b), en situaciones que requieren la recuperación controlada y el mantenimiento activo de la información, los individuos con alta capacidad de MO tienden a ser menos susceptibles a la distracción y disponen de procesos eficientes de discriminación y búsqueda en el sistema de memoria, que les permiten recuperar los segmentos relevantes de manera más rápida y exacta.

Schoonen, Snellings, Stevenson y Van Gelderen (2009) opinan que variables lingüísticas como la fluidez de vocabulario y la corrección gramatical "absorben" una gran cantidad de recursos cognitivos y comprometen la atención prestada a aspectos más globales (elaboración del contenido, revisión). Torrance y Galbraith (2006) apuntan que los escritores que logran suprimir ideas tangenciales y que son capaces de ignorar, aunque provisionalmente, una frase mal formulada, podrán llegar a concluir su frase antes de que se les olvide el mensaje que quieren expresar. Gass y Lee (2011), por su parte, mostraron empíricamente que la capacidad de la MO está estrechamente vinculada con la habilidad de separar diferentes aspectos de la lengua e ignorar o inhibir otros. La aplicación de control atencional y la inhibición de la interferencia y de los distractores constituyen funciones claves de la MO (Engle y otros, 1999; Kane y otros, 2007).

De acuerdo con lo expuesto, se podría inferir que los informantes del presente estudio con un span de $\mathrm{MO}$ alto consiguieron de modo más eficaz acceder, elegir y recuperar los segmentos adecuados y representaron fonológicamente $y$, tal vez, con mayor exactitud los cons- 
tituyentes de estos segmentos, lo cual probablemente facilitó su codificación ortográfica. En cambio, para aquellos aprendientes con un span de MO menor, la cantidad de procesos que debían llevarse a cabo seguramente abrumara sus recursos de memoria y condujera a mayores fluctuaciones en el nivel de atención prestado a los diferentes componentes de la producción escrita. Se puede especular que estos informantes se enfrentaron con mayores dificultades a la hora de acceder a su léxico mental y representar fonológicamente los segmentos que querían utilizar, equivocándose en la selección de las desinencias verbales, omitiendo una letra o una preposición. Todo ello probablemente dio lugar a un procesamiento lingüístico y monitorización del output deficientes y fragmentarios, a distracciones y a la activación de segmentos incorrectos, factores estos que acabaron afectando negativamente su actuación.

Jacoby (1978) sostuvo que es más fácil recuperar ítems que se han producido o utilizado en algún momento que los que simplemente se han leído o estudiado. Como explica el autor, cuando se repite un problema, el sujeto suele responder mediante el recuerdo de la solución de este problema y no por medio de operaciones más complejas, necesarias para abordar problemas y tareas nuevas. Por tanto, se podría suponer que, además de segmentos lingüísticos, el material gráfico potenció la recuperación de acciones, imágenes o soluciones específicas a problemas lingüísticos concretos (por ejemplo, recordar el contexto en el que se escuchó un segmento concreto, el manual en el que se había encontrado, consultado o estudiado, aquella composición o frase en la que tuvo que utilizarse). A pesar de que la actuación que se basa en soluciones obtenidas mediante el recuerdo puede no funcionar (Jacoby, 1978), para aquellos aprendientes con una mayor capacidad de MO acceder y recuperar estas soluciones podría ser un proceso más fácil e incluso podría convertirse en una estrategia compensatoria, sobre todo en aquellas situaciones en las que aumenta la carga de procesamiento y no se dispone de tiempo suficiente para elaborar respuestas nuevas o para buscar y analizar diferentes alternativas lingüísticas, como en el caso de la TI.

Sumado a lo anterior y teniendo en cuenta el perfil lingüístico del grupo meta, resulta oportuno ofrecer algunas puntualizaciones acerca de la implicación de la LM u otras LE en la escritura. Varios autores (Cook, 1999; Cumming, 2009; Manchón, Roca de Larios y Murphy, 2009; Ortega, 2009) argumentaron sobre el papel integral de la lengua dominante en los procesos de producción escrita en LE. La lengua dominante, que suele ser la LM, está constantemente presente durante el procesamiento lingüístico (Cook, 1999) y se convierte en un medio o estrategia (Ortega, 2009) que le sirve al aprendiente para probar su memoria, buscar e identificar problemas lingüísticos, y generar y evaluar alternativas a nivel léxico y conceptual (véase Manchón y otros, 2009: 114). De Bot (2004) señala que, si bien los segmentos de la LM presentarían supuestamente un nivel de activación más alto, para los aprendientes que poseen un alto dominio de varias LE la convergencia (fonológica, ortográfica, semántica) entre el código lingüístico de la(s) LE y la lengua meta podría dar lugar a una mayor activación de esta(s) LE en comparación con la LM. 
Los informantes del presente estudio, además de su LM, hablaban al menos una LE diferente al español y algunos habían estudiado hasta tres LE. Además, muchos de ellos se habían involucrado en el aprendizaje de LE, principalmente del inglés, desde edades muy tempranas (5 años) y durante un promedio de aproximadamente 8 años en el caso del inglés y 5 años para el francés. Es preciso señalar también que, en el contexto griego, la exposición al inglés suele empezar incluso antes de la edad de los 5 años y de la introducción del niño al sistema educativo, por ejemplo, a través de los programas televisivos (Masoura y Gathercole, 2005), lo cual hace de la cantidad de input recibido en este idioma una variable difícil de controlar, establecer o medir empíricamente.

El punto interesante en relación con el conocimiento de varios idiomas tiene que ver con el vínculo que se ha constatado entre ciertos fenómenos lingüísticos, como el cambio de código, y los procesos cognitivos y de MO. En concreto, se ha sostenido que los bilingües difieren de los monolingües en que los primeros cambian de un idioma al otro o incluso los utilizan al mismo tiempo (Cook, 1999; Cumming, 2009; Kutas, Moreno y Wicha, 2009). Las dos lenguas que se activan simultáneamente en la memoria de los bilingües pueden competir, converger, negociarse e influirse entre sí a nivel léxico, gramatical y fonológico (Kroll y Dussias, 2004). La necesidad de controlar las múltiples representaciones lingüísticas que se activan hace que los bilingües desarrollen y entrenen sus capacidades ejecutivas, necesarias para afrontar competiciones e interferencias durante el procesamiento lingüístico (Green, 1998; Szmalec, Brysbaert y Duyck, 2013). Asimismo, los resultados de estudios empíricos recientes han mostrado que el cambio de código no produce necesariamente un coste de procesamiento. Al contrario, parece que los diferentes idiomas hablados pueden cooperar durante la realización de tareas de comprensión lectora (Gullifer, Kroll y Dussias, 2013) y de expresión escrita (Tullock y Fernández-Villanueva, 2013).

En función de los planteamientos expuestos, y como se constató a partir del análisis del corpus de producciones escritas, los informantes del presente estudio recurrieron tanto a su LM como a otras LE que hablaban a fin de buscar determinados significados, corregir su producción o simplemente para poder continuar su discurso dentro de los límites de tiempo establecidos para la realización de las dos tareas. Es lógico pensar que para pasar de un código al otro es necesario, por un lado, contar con un espacio mental en el que se puedan guardar temporalmente y analizarse en tiempo real las representaciones lingüísticas procedentes de los diferentes códigos (LM, LE, lengua meta) y, por otro lado, aplicar control atencional e inhibitorio para que los ítems relevantes reciban mayor activación hasta su posterior transcripción. De acuerdo con estas líneas de argumentación inductiva, se podría sostener que los informantes del presente estudio con una mayor capacidad de MO lograron controlar las múltiples representaciones activadas y suprimieron la interferencia generada por los segmentos de los demás idiomas que hablaban, lo cual probablemente potenció la producción de un discurso escrito cualitativamente más adecuado y correcto. 
Considerando por separado la contribución de la MO (MST) a la realización de las Tı y T2, los modelos de regresión efectuados revelaron coeficientes de determinación moderados, siendo ligeramente mayores los correspondientes a la T1, que fue la tarea que se llevó a cabo bajo presión de tiempo. Según varios autores (Engle y otros, 1999; Robinson, 2003; Ilkowska y Engle, 2010), las diferencias individuales en la capacidad de la MO se manifiestan en situaciones y tareas conflictivas y estresantes, que presentan un alto grado de complejidad cognitiva y que requieren planificación, procesos de búsqueda y toma de decisiones para anular o debilitar respuestas habituales o automatizadas. Para afrontar situaciones como las descritas, el individuo se sirve tanto del procesamiento controlado, que es exacto pero lento, como del automático, que es propenso a errores (Ilkowska y Engle, 2010). La mayor cantidad de tiempo de la que dispusieron los informantes para realizar la T2 probablemente favoreció el procesamiento controlado. En cuanto a la T1, la necesidad de alternar entre procesamiento controlado y automático de manera más rápida quizás implicara un mayor esfuerzo o complejidad, de ahí que la intervención de la MO en dicha tarea fuera ligeramente mayor.

La necesidad de considerar la relación tiempo-esfuerzo no es nueva. Hace más de cuatro décadas Kahneman (1973) señalaba que la noción intuitiva de dificultad o la probabilidad de equivocación no son suficientes para determinar la cantidad de esfuerzo que requiere una tarea. Según Kahneman (1973), cuando se realizan tareas mentales, es imprescindible considerar la presión de tiempo que se impone tanto por las instrucciones proporcionadas como por las características de la tarea en sí. En cuanto al presente estudio, parece que restar a los informantes la oportunidad de planificar o revisar de modo más pormenorizado sus textos en el caso de la Tı supuso una carga cognitiva adicional en comparación con la T2, de ahí que se evidenciara una implicación ligeramente mayor de la MO en la realización de la T1.

Kellogg (2008) sostiene que la atención ejecutiva puede repartirse simultáneamente entre los diferentes procesos de la escritura, alternarse entre estos o dedicarse a unos a medida que se llevan a cabo otros procesos relativamente automáticos. No obstante, cuando el tiempo o el esfuerzo dedicado por el individuo resultan insuficientes, los procesos de escritura entran en competición [trade-offs] y requieren concentración sostenida para ejecutarse de manera eficiente (Kellogg, 1986, 2008). Dada la cantidad, la recursividad y el diferente grado de complejidad de estos procesos, se podría hablar de la escritura como una tarea, como mínimo, dual.

De acuerdo con Monsell (2003), el cambio de tarea [task switching] —que en el caso de la escritura puede entenderse como cambio de un proceso de escritura a otro- conduce a respuestas lentas y más propensas a errores. Este coste de cambio [switch cost] se reduciría no solo como resultado de una mayor capacidad de MO, sino también en correlación con el aumento del tiempo de preparación (preparation effect en palabras de Monsell) o del que se dispone para atender a los diferentes componentes de la escritura. Esta preparación probablemente fue favorecida por las condiciones bajo las cuales se llevó a cabo la T2. 
Ahora bien, para la correcta interpretación de los resultados conviene hacer algunas consideraciones adicionales. El hecho de que las diferencias en los coeficientes de determinación encontrados respecto a las T1EvGlobal y T2EvGlobal no fueran grandes podría indicar que la MO contribuye a la calidad de la expresión escrita en un grado relativamente estable, al menos cuando las tareas comparten ciertas características (el mismo género discursivo y bajas exigencias a nivel de conceptualización del mensaje, como fue el caso de las T1 y T2).

Alternativamente, se puede argumentar que las diferencias individuales en la capacidad de la MO se revelan incluso bajo condiciones favorables de actuación. Guará Tavares (2013), por ejemplo, sostuvo que la planificación potencia el comportamiento estratégico, que está vinculado con la MO, y facilita procesos de recuperación más eficientes por parte de aquellos aprendientes con alta capacidad de MO. Aunque la autora examinó el impacto de la planificación previa en el discurso oral, por lo que su argumentación puede no ser totalmente transferible al caso particular de la expresión escrita, se podría suponer que diferentes funciones de la MO se implican en la producción escrita bajo diferentes condiciones de actuación; por ejemplo, control atencional, resolución de conflictos e inhibición de interferencias cuando la tarea presenta un alto grado de complejidad cognitiva (T1) y comportamiento estratégico cuando la tarea implica niveles de complejidad manejables (T2).

Otro resultado de interés concierne al hecho de que las correlaciones halladas entre el span de MO y la producción escrita fueron significativas solo con respecto al MST. En esta prueba, el tiempo para la resolución de los problemas matemáticos y para la recuperación de los dígitos meta fue delimitado y estas dos tareas eran intercaladas. En cambio, en la realización del SST hubo algo de secuencialidad en las tareas primaria y secundaria (primero retener una serie de palabras y después producir con estas oraciones aceptables) y, aunque el tiempo para la formulación de oraciones fue también preestablecido, se comprobó que este fue suficiente o incluso excesivo. Parece que la mayor presión de tiempo en la ejecución del MST y la mayor interferencia generada por la necesidad de procesar, almacenar y verbalizar ítems de igual naturaleza y tipo (dígitos) constituyeron los rasgos determinantes de las correlaciones más altas que se obtuvieron entre dicha prueba y las T1EvGlobal y T2EvGlobal.

En lo que se refiere a la capacidad del componente fonológico de la MO, los resultados mostraron una correlación moderada entre dicha capacidad y la actuación de los informantes en la T2, aunque su contribución fue menor en comparación con el span de MO. Según Torrance y Galbraith (2006: 72), si se asume que la práctica de la escritura promueve un uso más eficaz de los recursos de la memoria y fomenta la automatización de algunos subprocesos de la escritura, a medida que el escritor madure, su capacidad de memoria fonológica desempeñará un papel secundario en el acto de escribir. Dada la alta consistencia fonología-ortografía del español y la amplia práctica de los hablantes nativos de griego con el sistema de escritura latino, podría afirmarse con cierto grado de seguridad que los informantes del presente 
estudio habían automatizado algunos subprocesos de la escritura; la ejecución de grafías sería un ejemplo. Sin embargo, esta interpretación explica solo parcialmente los resultados obtenidos, ya que, como se señaló anteriormente, la relación positiva entre la capacidad de la memoria fonológica y la actuación de los informantes se pudo constatar solo en la T2.

Se podría deducir que el tiempo dedicado a la escritura, sea este empleado para la planificación, lectura o revisión, influye de alguna manera en la relación entre la capacidad de la memoria fonológica y la actuación. En otras palabras, cuanto mayor sea ese tiempo, más probable es que el aprendiente recurra a procesos básicos de memoria, como la retención y el ensayo articulatorio de los segmentos que formarán parte de su output, procesos que se le atribuyen a la memoria fonológica. No obstante, es necesario subrayar que la diferencia en la magnitud de las correlaciones halladas entre la PRP y la T2EvGlobal ( $r=302$ ), por un lado, y el MST y la T2EvGlobal ( $r=356$ ), por otro, no fue muy grande. Este resultado podría indicar que, bajo condiciones favorables de actuación, las pruebas simples de MO resultan igual de eficaces para predecir la variabilidad en la escritura que las pruebas complejas de la misma. En todo caso, esta interpretación debe tomarse con precaución, ya que es posible que la PRP hubiera captado también algunos procesos atencionales o ejecutivos, especialmente a medida que aumentaba el nivel de dificultad de la prueba (niveles 5 y 6 de la PRP) (Unsworth y Engle, 2006, 2007a). La conclusión que se deriva de lo expuesto es que el componente fonológico de la MO no constituye aisladamente un factor determinante de los procesos de producción escrita en LE. Sin embargo, podría potencialmente ejercer una influencia positiva en la calidad del texto, siempre que lo permitan las condiciones de actuación.

En síntesis, los resultados del presente estudio remarcan la necesidad de seguir investigando la relación entre MO y expresión escrita desde la perspectiva de ASL a fin de poder determinar con mayor precisión cómo, hasta qué punto y en qué tipo de situaciones este mecanismo interviene en los procesos de escritura en una LE. Dado que este mecanismo comprende una multitud de almacenes y desempeña una multitud de funciones, sería de suma relevancia examinar en futuros estudios cómo el componente visoespacial de la MO o algunas funciones ejecutivas, como la inhibición y el cambio de la atención, influyen en los procesos de planificación, textualización y revisión, así como en aspectos lingüísticos concretos como la complejidad discursiva, de ideas y de contenido, la corrección o la complejidad de vocabulario. Asimismo, sería recomendable una réplica del presente estudio con una muestra de aprendientes más numerosa y homogénea respecto a ciertas características (cantidad de exposición al español, conocimiento de otras LE), con lo cual se podrían generalizar los resultados de manera más efectiva. Por último, es importante subrayar que cualquier predicción, inferencia o conclusión derivada del presente estudio se restringe a un único momento del proceso de aprendizaje de la LE. Es por ello que la investigación futura debería proporcionar datos de carácter longitudinal y, de esta manera, identificar los posibles beneficios de la MO en la producción escrita a largo plazo. 


\section{Bibliografía citada}

AndRADE, Jackie, 2001: "An Introduction to Working Memory" en Jackie Andrade (ed.): Working Memory in Perspective, Hove, UK: Psychology Press, 3-30.

Baddeley, Alan D., y Robert H. Logie, 1999: “Working Memory: The Multiple-Component Model” en Akira MiYake y Priti SHAH (eds.): Models of Working Memory: Mechanisms of Active Maintenance and Executive Control, New York, NY: Cambridge University Press, 28-61.

Brea-Spahn, María R., 2009: Spanish-Specific Patterns and Nonword Repetition Performance in English Language Learners. Unpublished Ph.D. thesis, University of South Florida.

Cook, Vivian, 1999: "Going Beyond the Native Speaker in Language Teaching”, TESOL Quarterly 33 (2), 185-209.

Cumming, Alister, 2009: "The Contribution of Studies of Foreign Language Writing to Research, Theories and Policies" en Rosa Manchón (ed.): Writing in Foreign Language Contexts: Learning, Teaching, and Research, Clevedon, UK: Multilingual Matters, 209-231.

De Bot, Kees, 2004: "The Multilingual Lexicon: Modelling Selection and Control", International Journal of Multilingualism 1 (1), 17-32.

Eguiluz Pacheco, Juan, y Ángel Eguiluz Pacheco, 2004: "La Evaluación de la expresión escrita” en Jesús Sánchez Lobato e Isabel Santos Gargallo (eds.): Vademécum para la formación de profesores: Enseñar Español como segunda lengua (L2)/ lengua extranjera(LE), Madrid, España: SGEL, 1005-1024.

Engle, Randall W., Judy Cantor y Julie J. Carullo, 1992: "Individual Differences in Working Memory and Comprehension: A Test of Four Hypothesis", Journal of Experimental Psychology: Learning, Memory, and Cognition 18 (5), 972-992.

Engle, Randall W., Michael J. Kane y Stephen W. Tuholski, 1999: "Individual Differences in Working Memory Capacity and What They Tell Us About Controlled Attention, General Fluid Intelligence, and Functions of the Prefrontal Cortex" en Akira Miyake y Priti SHAH (eds.): Models of working memory: Mechanisms of active maintenance and executive control, New York, NY: Cambridge University Press, 102-134.

FIELD, Andy, 2009 [2000]: Discovering Statistics Using SPSS, tercera edición, London, UK: SAGE.

Gass, Susan M., y Junkyu LeE, 2011: "Working Memory Capacity, Inhibitory Control, and Proficiency in a Second Language" en Monika S. ScHmid y Wander LowIE (eds.): Modeling Bilingualism: From Structure to Chaos, Amsterdam, The Netherlands: John Benjamins, 59-84.

Gathercole, Susan Elizabeth, 1995: "Is Nonword Repetition a Test of Phonological Memory or Long-term Knowledge? It All Depends on the Nonwords", Memory \& Cognition 23 (1), 83-94. 
GreEn, David W., 1998: "Mental Control of the Bilingual Lexico-Semantic System”, Bilingualism: Language and Cognition $1(2), 67-81$.

Guara TaVAREs, Maria da Glória, 2013: "Working Memory Capacity and L2 Speech Performance in Planned and Spontaneous Conditions: A Correlational Analysis", Trabalhos em Linguística Aplicada 52 (1), 9-29.

Gullifer, Jason W., Judith F. Kroll y Paola E. Dussias, 2013: "When Language Switching Has No Apparent Cost: Lexical Access in Sentence Context”, Frontiers in Psychology 4, Article 278.

Hallgren, Kevin A., 2012: "Computing Inter-Rater Reliability for Observational Data: An Overview and Tutorial”, Tutorials in Quantitative Methods for Psychology 8 (1), 23-34.

ILKowSKA, Malgorzata, y Randall W. Engle, 2010: "Working Memory Capacity and Self-regulation” en Rick H. Hoyle (ed.): Handbook of Personality and Self-regulation, West Sussex, UK: Wiley, 265-290.

JACOBY, Larry L., 1978: "On Interpreting the Effects of Repetition: Solving a Problem Versus Remembering a Solution”, Journal of Verbal Learning and Verbal Behavior 17 (6), 649-667.

Kahneman, Daniel. 1973: Attention and Effort. Englewood Cliffs, NJ: Prentice-Hall.

Kane, Michael J., Andrew R. A. Conway, David Zachary Hambrick y Randall W. Engle, 2007: "Variation in Working Memory Capacity as Variation in Executive Attention and Control" en Andrew E. A. Conway, Chris Jarrold, Michael J. Kane, Akira Miyake y John Towse (eds.): Variation in Working Memory, New York, NY: Oxford University Press, 21-48.

Kellogg, Ronald T., 1986: “Designing Idea Processors for Document Composition”, Behavior Research Methods, Instruments, \& Computers 18 (2), 118-128.

KellogG, Ronald T., 2008: “Training Writing Skills: A Cognitive Developmental Perspective”, Journal of Writing Research 1 (1), 1-26.

Kormos, Judit, 2012: "The Role of Individual Differences in L2 Writing”, Journal of Second Language Writing 21 (4), 390-403.

Kroll, Judith F., y Paola E. Dussias, 2004: "The Comprehension of Words and Sentences in Two Languages” en Tej K. Bhatia y William C. Ritchie (eds.): The Handbook of Bilingualism, Malden, MA: Blackwell Publishing, 169-200.

Kutas, Marta, Eva M. Moreno y Nicole Yvonne Wicha, 2009: "Code Switching and the Brain" en Barbara E. Bullock y Almeida Jacqueline Toribı (eds.): The Cambridge Handbook of Linguistic Code-Switching, Cambridge: Cambridge University Press, 289-306.

Kutner, Michael H., Christopher J. Nachtsheim, John Neter y William LI, 2005 [1974]: Applied Linear Statistical Models, quinta edición, New York, NY: McGraw-Hill. 
Manchón, Rosa M., 2009: "Introduction: Broadening the Perspective of L2 Writing Scholarship: The Contribution of Research on Foreign Language Writing" en Rosa M. Manchón (ed.): Writing in Foreign Language Contexts: Learning, Teaching, and Research, Clevedon, UK: Multilingual Matters, 1-19.

Manchón, Rosa M., Julio Roca de Larios y Liz Murphy, 2009: "The Temporal Dimension and ProblemSolving Nature of Foreign Language Composing Processes. Implications for Theory" en Rosa M. MAnchón (ed.): Writing in Foreign Language Contexts: Learning, Teaching, and Research, Clevedon, UK: Multilingual Matters, 102-129.

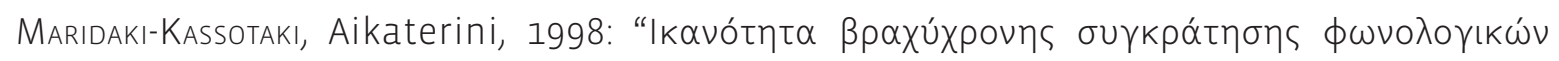

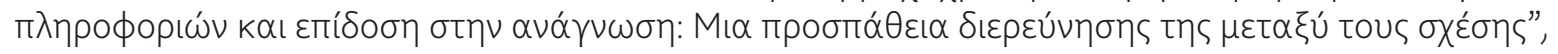
Psychology: The Journal of the Hellenic Psychological Society 5 (1), 44-52.

Masoura, Elvira V., y Susan Elizabeth Gathercole, 2005: "Contrasting contributions of phonological short-term memory and long-term knowledge to vocabulary learning in a foreign language" Memory 13 (3-4), 422-429.

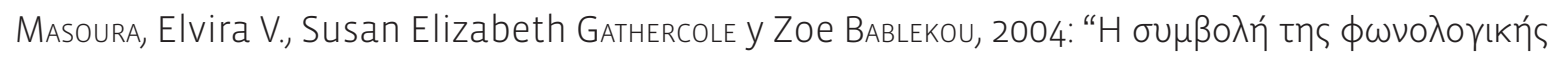

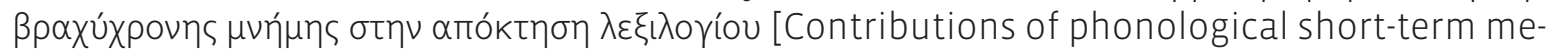
mory to vocabulary acquisition]", Psychology: The Journal of the Hellenic Psychological Society 11 (3), 341-355.

Mavrou, Irini, 2017: "Memoria operativa y expresión escrita: Un área de investigación pendiente en el campo de la Adquisición de Segundas Lenguas", Porta Linguarum 27, 171-183.

MAVRou, Irini, 2018: "Memoria operativa y trade-offs entre los constructos de complejidad, precisión y fluidez”, Moderna Språk 112 (1), 106-140.

Mavrou, Irini, y Claudia Mariella Villar, 2017: "La influencia de la manipulación del tiempo en la complejidad, corrección y fluidez lingüísticas de tareas escritas en lengua extranjera”, Sintagma 29, 7-26.

Mizera, Gregory J., 2006: Working Memory and L2 Oral Fluency. Unpublished Ph.D. thesis, University of Pittsburgh.

MonselL, Stephen, 2003: "Task Switching”, TRENDS in Cognitive Sciences 7 (3), 134-140.

Mota, Mailce Borges, 2003: "Working Memory Capacity and Fluency, Accuracy, Complexity, and Lexical Density in L2 Speech Production”, Fragmentos 24, 69-104.

ORtegA, Lourdes, 2009: "Studying Writing Across EFL Contexts: Looking Back and Moving Forward" en Rosa M. Manchón (ed.): Writing in Foreign Language Contexts: Learning, Teaching, and Research, Clevedon, UK: Multilingual Matters, 232-255. 
PrebiancA, Gicele V. V., y Raquel D’Ely, 2008: “EFL Speaking and Individual Differences in Working Memory Capacity: Grammatical Complexity and Weighted Lexical Density in the Oral Production of Beginners", Signótica 20 (2), 335-366.

RoBInson, Peter, 2001: "Task Complexity, Cognitive Resources, and Syllabus Design: A Triadic Framework for Examining Task Influences on SLA" en Peter RoBInson (ed.): Cognition and Second Language Instruction, Cambridge: Cambridge University Press, 287-318.

Robinson, Peter, 2003: "The Cognition Hypothesis, Task Design, and Adult Task-based Language Learning”, Second Language Studies 21 (2), 45-105.

Schoonen, Rob, Patrick Snellings, Marie Stevenson y Amos van Gelderen, 2009: "Towards a Blueprint of the Foreign Language Writer: The Linguistic and Cognitive Demands of Foreign Language Writing" en Rosa M. Manchón (ed.): Writing in Foreign Language Contexts: Learning, Teaching, and Research, Clevedon, UK: Multilingual Matters, 77-101.

Shahnazari-Dorcheh, Mohammadtaghi, y Saeed Roshan, 2012: "Developing a Non-Language Related Span Test for the Use in Language-Specific and Cross-Language Studies", English Language Teaching 5 (11), 104-110.

Silva, Tony, Ilona Lekı y Joan Carson, 1997: "Broadening the Perspective of Mainstream Composition Studies. Some Thoughts from the Disciplinary Margins”, Written Communication 14 (3), 398-428.

Skehan, Peter, y Pauline Foster, 2001: “Cognition and Tasks" en Peter Robinson (ed.): Cognition and Second Language Instruction, Cambridge: Cambridge University Press, 181-205.

Stevens, James P., 1984: "Outliers and Influential Data Points in Regression Analysis", Psychological Bulletin 95 (2), 334-344.

Streiner, David L., 2003: "Starting at the Beginning: An Introduction to Coefficient Alpha and Internal Consistency", Journal of Personality Assessment 80 (1), 99-103.

Szmalec, Arnaud, Marc Brysbaert y Wouter Duyck, 2013: "Working Memory and (Second) Language Processing" en Jeanette AltarRIBA y Ludmila Isurin (eds.): Memory, Language, and Bilingualism: Theoretical and Applied Approaches, New York, NY: Cambridge University Press, 74-94.

Torrance, Mark, y David Galbraith, 2006: "The Processing Demands of Writing” en Charles A. MacArthur, Steve Graham y Jill Fitzgerald (eds.): Handbook of Writing Research, New York, NY: Guilford Press, 67-80.

Tullock, Brandon D., y Marta Fernández-Villanueva, 2013: "The Role of Previously Learned Languages in the Thought Processes of Multilingual Writers at the Deutsche Schule Barcelona", Research in the Teaching of English 47 (4), 420-441. 
TURner, Marilyn L., y Randall W. EngLe, 1989: “Is Working Memory Capacity Task Dependent?”, Journal of Memory and Language 28 (2), 127-154.

Unsworth, Nash, y Randall W. Engle, 2006: "Simple and Complex Memory Spans and their Relation to Fluid Abilities: Evidence from List-Length Effects", Journal of Memory and Language 54 (1), 68-80.

Unsworth, Nash, y Randall W. Engle, 2007a: "On the Division of Short-Term and Working Memory: An Examination of Simple and Complex Span and their Relation to Higher Order Abilities", Psychological Bulletin 133 (6), 1038-1066.

Unsworth, Nash, y Randall W. Engle, 2007b: "The Nature of Individual Differences in Working Memory Capacity: Active Maintenance in Primary Memory and Controlled Search from Secondary Memory", Psychological Review 114 (1), 104-132.

Wang, Ping, 2009: "The Inter-Rater Reliability in Scoring Composition”, English Language Teaching 2 (3), 39-43. 